\title{
The Matrix Pencil method applied to smart monitoring and radar
}

\author{
K. El Khamlichi Drissi ${ }^{1,2} \&$ D. Poljak ${ }^{3}$ \\ ${ }^{1}$ Clermont Université, Université Blaise Pascal, Institut Pascal, France \\ ${ }^{2}$ CNRS, UMR 6602, France \\ ${ }^{3}$ FESB, University of Split, Croatia
}

\begin{abstract}
The paper deals with the Matrix Pencil method, an efficient numerical method to identify deterministic signals based on either simulation or measurement. The method is usually referred to as a high-resolution technique as it is capable of identifying non-stationary signals, even in the presence of numerical or measurement noise, respectively. The basis functions are complex exponentials with a damping factor. Those functions are rather appropriate to identify electromagnetic waves or distributed currents with an optimal number of elements. The method is applied either in time domain, in frequency domain or in space domain allowing one to identify the original signal by means of a limited number of singular values, poles and residues.
\end{abstract}

Keywords: Matrix-Pencil method, NIALM, signature, radar, classification.

\section{Introduction}

This paper focuses on the usage of the Matrix Pencil method for estimating parameters of exponentially damped sinusoids for different domains; time domain, frequency domain or space domain.

The applications of interest in this paper are; non-intrusive identification and load monitoring in residential areas, a radiated electromagnetic field by a multiconductor transmission line system and also UWB radar for communication, localization and/or target identification.

The identification domain is chosen according to the simplicity of representation and to the limitation of a set of exponentials. It can be time domain, frequency domain or space domain. 
In the time domain, the responses are in real axis (NIALM applications and the case of UWB radar).

In frequency domain and in space domain, the responses are in the complex plane (radiated field and the case of UWB radar as well).

\section{The Matrix Pencil method}

The Matrix Pencil method is based on an adaptive subspace analysis and the exponentially damped sinusoids model. Subspace based signal analysis involves splitting the observations into a set of desired $\left(\mathrm{h}_{\mathrm{r}}\right)$ and a set of disturbing components $\left(b_{r}\right)$, which can be viewed in terms of signal and noise subspaces. This approach has been widely studied in the fields of adaptive filtering, source localization, or parameter estimation [1]. The eigenvalue decomposition (EVD) and the singular value decomposition (SVD) are commonly used in subspace estimation. After the sampling procedure, the observed response can be written as follows [2]:

$$
\mathrm{y}_{\mathrm{r}}\left(\mathrm{kT}_{\mathrm{s}}\right)=\mathrm{h}_{\mathrm{r}}\left(\mathrm{kT}_{\mathrm{s}}\right)+\mathrm{b}_{\mathrm{r}}\left(\mathrm{kT}_{\mathrm{s}}\right) \approx \sum_{\mathrm{r}=1}^{\mathrm{N}} \mathrm{R}_{\mathrm{r}} \mathrm{z}_{\mathrm{r}}^{\mathrm{k}}+\mathrm{b}_{\mathrm{r}}\left(\mathrm{kT}_{\mathrm{s}}\right)
$$

where $\mathrm{T}_{\mathrm{s}}$ is the sampling period, $\mathrm{h}_{\mathrm{r}}\left(\mathrm{kT}_{\mathrm{s}}\right)$ is the signal, and $\mathrm{b}_{\mathrm{r}}\left(\mathrm{kT}_{\mathrm{s}}\right)$ is the noise, with:

$$
\begin{gathered}
R_{r}=\left|a_{r}\right| e^{j \varphi_{r}} \quad r=1,2, \ldots, M \\
z_{r}=e^{S_{r} T_{s}}=e^{\left(\alpha_{r}+j \omega_{r}\right) T_{s}} \quad r=1,2, \ldots, M .
\end{gathered}
$$

The MPM is performed in a few steps:

First, Hankel's matrix using singular value decomposition (SVD) is calculated to determine the number of poles $M$ and the value $z_{r}$ :

$$
\mathrm{Y}_{\mathrm{r}}=\left(\begin{array}{cccc}
\mathrm{y}_{\mathrm{r}}(0) & \mathrm{y}_{\mathrm{r}}(1) & \cdots & \mathrm{y}_{\mathrm{r}}(\mathrm{L}) \\
\mathrm{y}_{\mathrm{r}}(1) & \mathrm{y}_{\mathrm{r}}(2) & \cdots & \mathrm{y}_{\mathrm{r}}(\mathrm{L}+1) \\
\vdots & \vdots & \vdots & \vdots \\
\mathrm{y}_{\mathrm{r}}(\mathrm{K}-\mathrm{L}-1) & \mathrm{y}_{\mathrm{r}}(\mathrm{K}-\mathrm{L}) & \cdots & \mathrm{y}_{\mathrm{r}}(\mathrm{K}-1)
\end{array}\right)
$$

where $Y_{r}$ is of dimensions $(K-L) \times(L+1)$ and $L$ is the Pencil parameter. The choice of $\mathrm{L}$ is important to reduce numerical noises. The best choice is shown to be [3]:

$$
\frac{\mathrm{K}}{2} \leq \mathrm{L} \leq \frac{2 \mathrm{~K}}{3}
$$

Next, a singular value decomposition of the matrix is carried out as:

$$
[\mathrm{Y}]=[\mathrm{U}] \cdot[\Sigma] \cdot[\mathrm{V}]^{\mathrm{H}}
$$

where $[\mathrm{U}]$ and $[\mathrm{V}]$ are unitary matrix of eigenvectors, while $[\Sigma]$ is a diagonal matrix of singular values $\sigma_{\mathrm{i}}$. The number of significant poles $\mathrm{M}$ should be estimated from the ratio of singular values to the largest one: 


$$
\frac{\sigma_{\mathrm{M}}}{\sigma_{1}} \approx 10^{-\mathrm{p}}
$$

where $p$ is the number of significant decimal digits in the data. The value of $M$ should satisfy the dual condition:

$$
\mathrm{M} \leq \mathrm{L} \leq \mathrm{K}-\mathrm{M}
$$

Next, the filtered matrix $\left[\mathrm{V}_{\mathrm{p}}\right]$, corresponding to the first $\mathrm{M}$ vectors of $[\mathrm{V}]$ is considered:

$$
\left[\mathrm{V}_{\mathrm{p}}\right]=\left[\mathrm{v}_{1}, \mathrm{v}_{2}, \ldots, \mathrm{v}_{\mathrm{M}}\right]
$$

From $\left[\mathrm{V}_{\mathrm{p}}\right]$ we generate $\left[\mathrm{V}_{\mathrm{p} 1}\right]$ by deleting the last row of $\left[\mathrm{V}_{\mathrm{p}}\right]$, and $\left[\mathrm{V}_{\mathrm{p} 2}\right]$ by deleting the first row of $\left[\mathrm{V}_{\mathrm{p}}\right]$. The eigenvalue of lower matrix rank $\left\{\left[\mathrm{V}_{\mathrm{p} 2}\right]^{\mathrm{H}}-\right.$ $\left.\lambda\left[\mathrm{V}_{\mathrm{p} 1}\right]\right\}$ provides the estimation of $\mathrm{Z}_{\mathrm{n}}$.

Once $M$ and $\mathrm{Z}_{\mathrm{n}}$ are known, the residues $\mathrm{R}_{\mathrm{n}}$ are obtained by using the following least square equation:

$$
\left(\begin{array}{c}
\mathrm{y}(0) \\
\mathrm{y}(1) \\
\vdots \\
\mathrm{y}(\mathrm{K}-1)
\end{array}\right)=\left(\begin{array}{cccc}
1 & 1 & \cdots & 1 \\
\mathrm{z}_{1} & \mathrm{z}_{2} & \cdots & \mathrm{z}_{\mathrm{N}} \\
\vdots & \vdots & \vdots & \vdots \\
\mathrm{z}_{1}^{(\mathrm{K}-1)} & \mathrm{z}_{2}^{(\mathrm{K}-1)} & \cdots & \mathrm{z}_{\mathrm{M}}^{(\mathrm{K}-1)}
\end{array}\right)\left(\begin{array}{c}
\mathrm{R}_{1} \\
\mathrm{R}_{2} \\
\vdots \\
\mathrm{R}_{\mathrm{M}}
\end{array}\right)
$$

When applying MPMTD, the database is filled in with damping factors (real part of poles) and positive pulsation of resonances (imaginary part of poles). However, when applying it on the frequency signal, the database is filled in with all real and imaginary parts of the poles, because they are not conjugate poles.

To illustrate the efficiency of the method, the application of the procedure to Hankel function $H_{0}$ in complex plane is carried out.

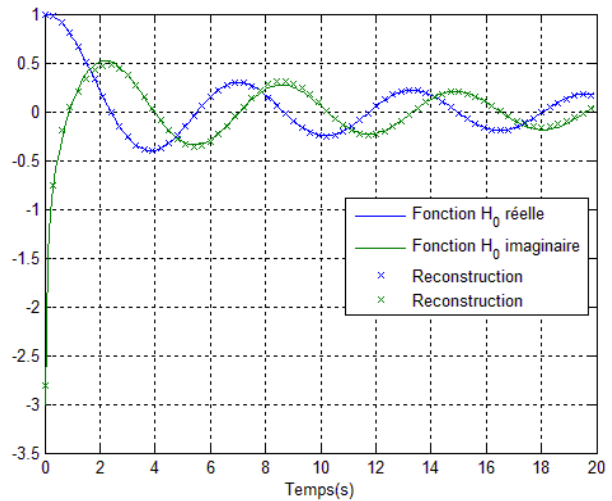

Figure 1: Hankel function identification.

For the real part of $H_{0}$, the set of poles are $s_{1,2}=-0.5838 \pm$ $0.7559 j$ and $s_{3,4}=-0.0424 \pm 0.9967 j$, the set of residues are $R_{1,2}=$ $0.3478 \mp 0.1480 j$ and $R_{3,4}=0.14764 \mp 0.1316 j$. 
For the imaginary part of $H_{0}$, the set of poles are $s_{1}=-5.1401, s_{2}=$ -0.1354 and $s_{3,4}=-0.0667 \pm 1.0013 j$, the set of residues are $R_{1}=$ $-2.4554, R_{2}=-+0.0271$ and $R_{3,4}=-0.1936 \pm 0.1867 j$.

\section{Application in the time domain: electrical load monitoring}

It is an important topic to address a larger monitoring system called smart metering. The MP method in the time domain to treat the problem of non intrusive appliance load monitoring (NIALM) [4] is introduced, the main goal is to monitor load appliances in residential home and without intrusion. This technique takes the advantage from the transient and steady state analysis to improve monitoring efficiency. In particular, it allows us to extract active and reactive power for fundamental and harmonic frequencies. This new approach has been integrated into an energy meter with the collaboration of EDF, Landis + Gyr and the Institut Pascal. Identification signal based on poles and residues is used not only for slow or fast transients, but also for the steady state. The result is successful and has been patented both in Europe and overseas [5]. Actually, an implementation in smart meters is in progress.

Fixed edge detection identifies changes in steady-state poles and residues domain. If the transition is larger than a defined threshold, an event is declared. The corresponding time is stored. Actually, load classification is related to its cycle from ON state to OFF state. A database containing loads signatures is correlated to extracted signature. If the new signature is not in the database, it will be automatically added to the database of the electrical loads.

Energy consumption is calculated from the information obtained from the load-classification module (poles and residues, load turn ON and load turn OFF). Energy consumption can be split into four groups of loads: lighting, water heating, heating and "other appliances".

The current and voltage are divided into the sliding window of width $\Delta \mathrm{t}$ and are treated by the Matrix Pencil method. Generally, the supply voltage is a periodic pulse signal with period $\mathrm{T}$ and pulsation $\omega$. The duration of each window $\Delta \mathrm{t}$ may be set equal to the period $\mathrm{T}$. This choice is similar to classical energy meters treating periodic signals. Each window $\Delta \mathrm{t}$ of voltage is represented by $M^{\prime}$ couples of poles and residues $\left(R_{j}^{\prime}, S_{j}^{\prime}\right.$ with $\left.1 \leq j \leq M^{\prime}\right)$ and each window of current is represented by $M$ couples of poles and residues $\left(R_{i}, S_{i}\right.$ with $1 \leq \mathrm{i} \leq \mathrm{M})$.

The active power is expressed as the sum of the contribution of current and voltage.

$$
P=\sum_{i=1}^{M} \sum_{j=1}^{M \prime} \frac{R_{i} R_{j}}{2} e^{\left(S_{i}+S_{j}^{\prime}\right)(\Delta t / 2)} \cdot \sinh c\left(\left(S_{i}+S_{j}^{\prime}\right) \frac{\Delta t}{2}\right)
$$

\subsection{Experimental results}

An application of this method is carried out by using the smart meter which is described elsewhere [6]. Several campaigns of measurement were carried out in the home automation located at the research center of EDF. The objective was to 
record load signatures, to assess the efficiency of our digital platform and to prove its ability to monitor each of the existing electric load. The measurement results were compared to simulations obtained by Matlab software. The results agree satisfactorily. The appliances are monitored individually and in combinatorial way.

The treated example is a water heater load; the input current during the experiments is given in Figure 2. The window duration of treatment is $600 \mathrm{~s}$.

A zoom view on six periods of the input current is represented in Figure 3.

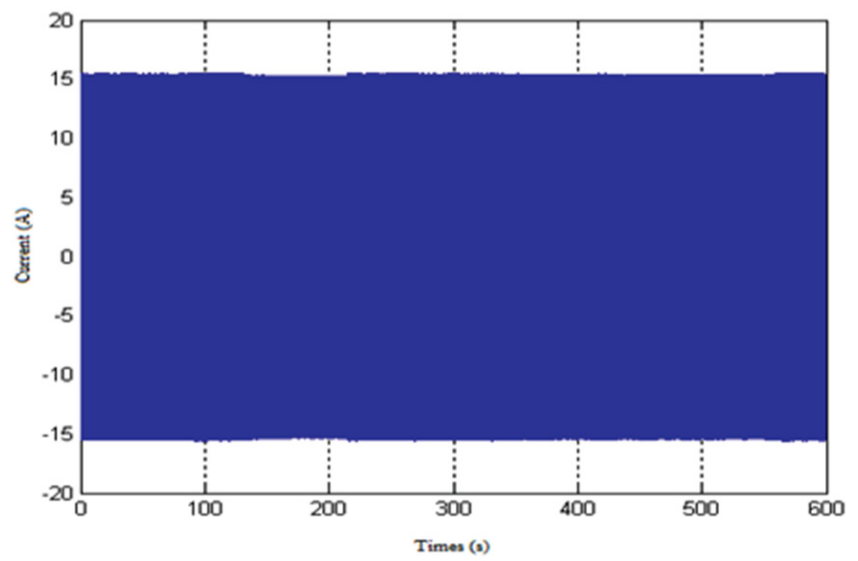

Figure 2: Current of the water heater.

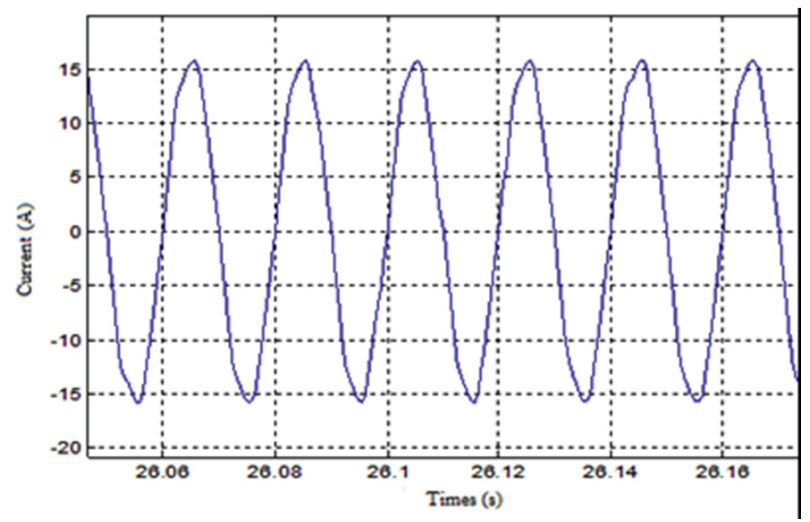

Figure 3: Zoom on the water heating input current.

\subsection{Load identification}

The input current waveforms may be correctly approximated by a sine wave with a period of $20 \mathrm{~ms}$. 

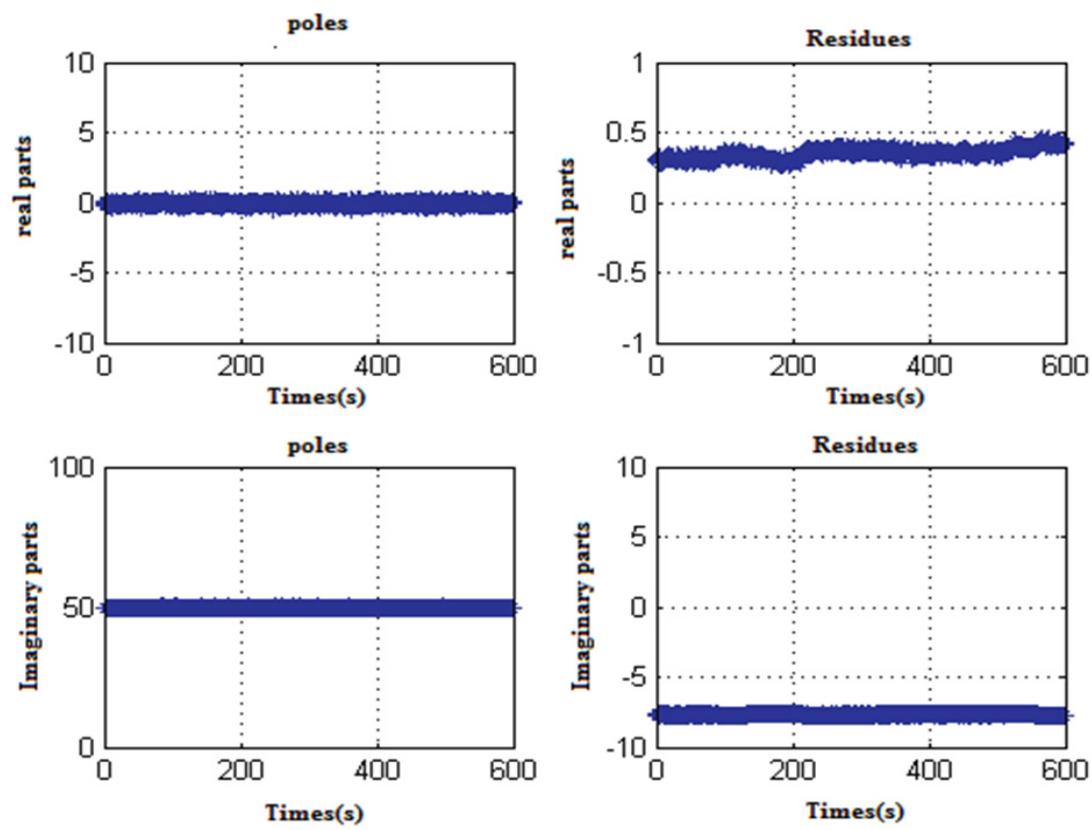

Figure 4: "Matrix Pencil" applied to water heater for load monitoring.

This result clearly shows that the load can be approximated to a resistive one. Consequently, theoretical approach expresses poles and residues in that case:

$$
S=\frac{\alpha+j \omega}{2 \pi}=0+50 j \text { and } R=\frac{E \sqrt{2}}{2 r j} \approx 0-7,7 j
$$

By using theoretical results and experimental values, heating water can be classified as a resistive load of $r=21 \Omega$.

Figure 4 shows the poles and residues variation in time domain. The sampling period is equal to the window width, i.e. $20 \mathrm{~ms}$.

The imaginary part of the pole is constant and equal to $50 \mathrm{~Hz}$, it means that the input current is identified by its fundamental.

The imaginary part of the residue is also constant and equal to 7,7 $\mathrm{A}$. The real part, which is negligible compared to imaginary part, represents the signal distortion.

We have generalized our approach of load identification and monitoring. We have tested it in a home automation and applied to several loads according to specific scenarios. Poles and residues extracted from our digital platform are correlated to a predetermined database. If the correlation is correct, the electrical load is identified and classified by group of appliances. Otherwise, the load is classified in group "others".

The corresponding energy is measured from poles and residues and added to each group of appliances.

The total energy is compared to the one given by the basic energy meter and agrees satisfactorily. 


\section{Application in frequency domain: radar target identification}

It is well known that when a radar target is excited by electromagnetic waves, its time domain response carries the signature of the target [7, 8]. The complete response of such a target consists of two parts. The first part is known as the early-time scattered response, which appears when the excitation wave front goes through the target. The second part, the late-time scattered response, appears when the excitation wave front moves beyond the target. UWB radar can be used in intelligent vehicles for communication, localization and/or target identification. This part deals with the target identification by using the Matrix Pencil method in the frequency domain (MPMFD) and in the complex plane. The method is applied to UWB backscattered signal from three canonical targets (thin wire, sphere, and cylinder). When a target is illuminated by ultra wideband signals, the scattered transient response in the time domain consists of an impulsive waveform part (early time) and a damped waveform (late time). The early time response arises from the direct reflection of the incident wave on the object surface. In the late time, the oscillating part arises from resonance phenomena of the target. The resonances can be separated into internal and external modes [9]. The internal resonances are caused by the internal waves experiencing multiple internal reflections, whereas the external resonances are caused by the surface creeping waves. In the case where targets are perfect conductors, resonances occur outside the object and correspond only to external modes.

In the frequency domain, the scattered fields $\mathrm{E}_{v}(\omega, \mathrm{r})$ can be expressed as [10]:

$$
E_{v}(\omega, r)=\sum_{n=1}^{\infty} A_{v n}(\omega, r) \exp \left(-j \omega t_{n}\right)
$$

where $v$ is any scattering field component of an orthogonal coordinate system (polarization), $\omega$ is the angular frequency, $A_{v n}(\omega, r)$ is the complex and frequency-dependent amplitude of the $n^{\text {th }}$ scattering center depending on the scattering mechanism, $r$ is the far-field position, $t_{n}$ is the time delay between the observer and the $n^{\text {th }}$ scattering center. The time dependence $\exp (j \omega t)$ and the $v$ are dropped for convenience throughout.

The following approximation [11] is used:

$$
A_{n}(\omega, r) \approx a_{n}(r) e^{\left(\gamma_{n} \omega\right)}
$$

where $a_{n}(r)$ is the amplitude, and $\gamma_{n}$ is the phase which provides an approximate match between $A_{n}(\omega, r)$ and the exponential model. This approximation can only be satisfied over a relatively narrow bandwidth. In our identification method, we divide $E(\omega, r)$ by the incident field. Therefore, after using (13) in (12), and the sampling procedure, the frequency response $Y\left(k \omega_{s}\right)$ will be expressed as:

$$
Y\left(k \omega_{s}\right) \approx \sum_{n=1}^{M_{f}} \hat{a}_{n}(r) e^{\left(\gamma_{n} k \omega_{s}-j k \omega_{s} t_{n}\right)}+n\left(k \omega_{s}\right) \approx \sum_{n=1}^{M_{f}} \hat{a}_{n}(r) x_{n}+n\left(k \omega_{s}\right)
$$

where $\omega_{s}$ is the frequency sampling, $N_{f}$ is the number of frequency samples, $M_{f}$ is the number of measurable wave fronts, $\hat{a}_{n}(r)$ is the complex amplitude, and $n\left(k \omega_{s}\right)$ is the additive noise, with

$$
x_{n}=e^{\left(\gamma_{n} k \omega_{s}-j k \omega_{s} t_{n}\right)}
$$


In frequency domain, the Matrix Pencil method could be applied to extract the features of different targets. The field scattered from the object is obtained from ultra wide band radar acting as both transmitter and receiver.

\subsection{Simulation results}

To compute the free-space backscattered fields of the targets, electromagnetic commercial tool (FEKO) is used [12]. A Gaussian pulse is used as an excitation plane wave; the incidence is normal. In the time domain, the expression of the Gaussian pulse waveform is given by:

$$
g(t)=A e^{-\left(\frac{t-t_{0}}{\sigma}\right)^{2}}
$$

where A stands for the amplitude and $\sigma$ for the width of the Gaussian pulse. In our simulations, we have set $\mathrm{A}=1, \mathrm{t}_{0}=10 \mathrm{~ns}$, and $\sigma=0.45455 \cdot 10^{-9} \mathrm{~s}^{-1}$.

$\sigma$ is chosen such that [12]: the upper frequency $f_{\max } \approx \frac{4}{\pi \sigma} \sqrt{\ln \sqrt{2}} \approx$ 1.7 GHz. Making a compromise between the calculation times and having the radiation of the targets in resonance and optical regions choose this frequency. Note that the frequency band used in our work for target classification is relatively narrow so that the approximation can be used (13).

With the TIME-FEKO program, electromagnetic fields are given in the time domain. It is based on the FEKO program (that does the relevant calculations in the frequency domain) and an IFFT algorithm that transforms the data to the time domain. The number of samples is chosen to be at 256 .

The backscattered field is given in the time domain without considering the factor $\frac{e^{-j k R}}{R}$.

To apply the MPMFD, we have worked with the frequency response, which can be obtained from the temporal response by transforming it to the frequency domain by means of the fast Fourier transformed (FFT) technique and dividing it by the FFT of the Gaussian incident pulse.

Simulations are conducted by using three metallic canonical objects: thin wire, sphere and cylinder. A database contains features extracted by MPMFD, The accuracy of the classification in three different classifiers [13]: Naïve Bayes (NB), K-Nearest Neighbour (K-NN), and Support Vector Machine (SVM) has been determined. We present the features extraction in frequency domain applied to thin wire, sphere and cylinder.

In Figure 5, we can see the excellent agreement between the scattered signal from canonical object and the reconstructed one by using MPM. In comparison to the contribution of the signal in the frequency domain, which takes the early time into consideration (Figure 5). The late-time signature for most realistic targets is usually very weak and heavily corrupted by noise.

Table 1 shows the extracted features with MPMFD from the same targets: the thin wire $(1=3 \mathrm{~m}, \mathrm{r}=0.0025 \mathrm{~m})$, the sphere $(\mathrm{r}=0.3 \mathrm{~m})$, and the cylinder $(\mathrm{l}=0.6 \mathrm{~m}, \mathrm{r}=0.2 \mathrm{~m})$. 


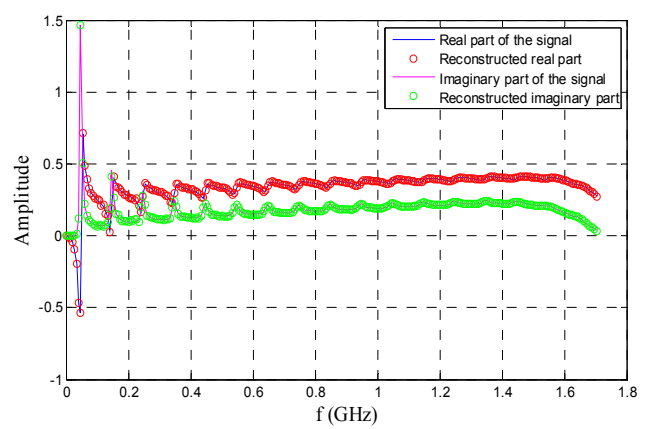

(a) Thin wire (length $=3 \mathrm{~m}$ and radius $=0.0025 \mathrm{~m})$.

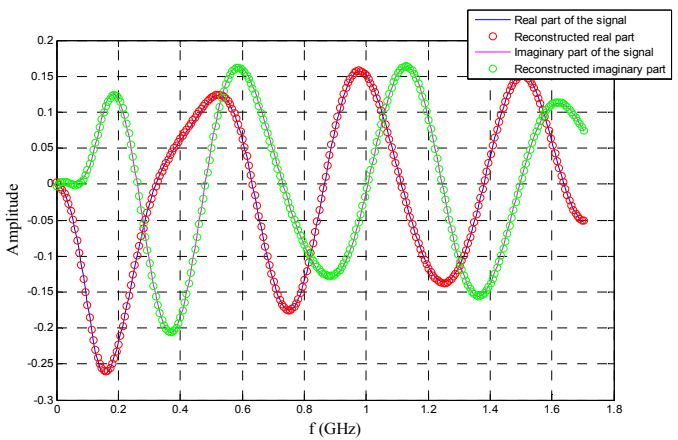

(b) Sphere (radius $=0.3 \mathrm{~m})$.

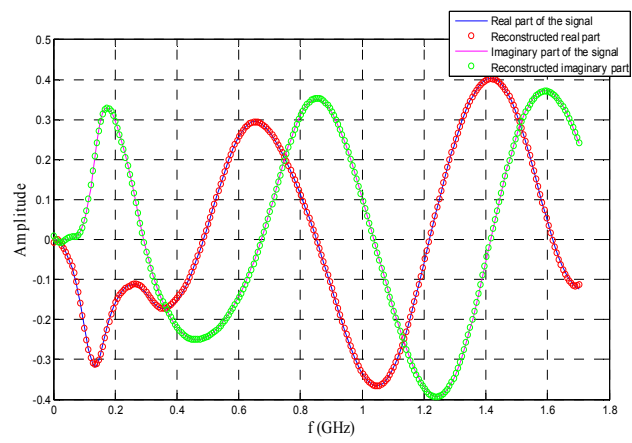

(c) Cylinder (length $=0.6 \mathrm{~m}$ and radius $=0.2 \mathrm{~m}$ ).

Figure 5: Simulated signal compared with a reconstructed signal by MPMFD.

With the poles shown in table 1, the real and imaginary parts of the signal have been reconstructed in figure 5 for the three objects. The obtained results agree satisfactorily with the simulated signal.

In frequency domain, all the physical characteristics of the objects are taking into account. The real and imaginary parts of the signals are used to identify the objects. 
Table 1: $\quad$ Features extracted by MPMFD from a thin wire $(\mathrm{l}=3 \mathrm{~m})$, a sphere $(\mathrm{r}=0.3 \mathrm{~m})$, and a cylinder $(\mathrm{l}=0.6 \mathrm{~m}, \mathrm{r}=0.2 \mathrm{~m})($ poles $\times 108)$.

\begin{tabular}{|l|c|l|l|}
\hline & Thin wire & \multicolumn{1}{c|}{ Sphere } & \multicolumn{1}{c|}{ Cylinder } \\
\hline $\mathbf{P}_{\mathbf{1}}$ & $-5.096-43.80 \mathrm{j}$ & $-0.60-2.970 \mathrm{j}$ & $-0.642-3.443 \mathrm{j}$ \\
\hline $\mathbf{P}_{\mathbf{2}}$ & $-4.678-33.57 \mathrm{j}$ & $-0.150-2.046 \mathrm{j}$ & $-0.184-1.994 \mathrm{j}$ \\
\hline $\mathbf{P}_{\mathbf{3}}$ & $-2.278-24.07 \mathrm{j}$ & $-1.013-1.108 \mathrm{j}$ & $-0.584-1.122 \mathrm{j}$ \\
\hline $\mathbf{P}_{\mathbf{4}}$ & $-1.169-19.47 \mathrm{j}$ & $-0.740+0.884 \mathrm{j}$ & $0.093-0.583 \mathrm{j}$ \\
\hline $\mathbf{P}_{\mathbf{5}}$ & $-0.426-12.61 \mathrm{j}$ & $-0.002+1.242 \mathrm{j}$ & $0.039+0.793 \mathrm{j}$ \\
\hline $\mathbf{P}_{\mathbf{6}}$ & $-10.80-7.60 \mathrm{j}$ & 0 & $0.003+1.014 \mathrm{j}$ \\
\hline $\mathbf{P}_{\mathbf{7}}$ & $-4.173-6.851 \mathrm{j}$ & 0 & 0 \\
\hline $\mathbf{P}_{\mathbf{8}}$ & $-0.229-6.294 \mathrm{j}$ & 0 & 0 \\
\hline $\mathbf{P}_{\mathbf{9}}$ & $0.021-0.002 \mathrm{j}$ & 0 & 0 \\
\hline $\mathbf{P}_{\mathbf{1 0}}$ & $-0.292+0.534 \mathrm{j}$ & 0 & 0 \\
\hline $\mathbf{P}_{\mathbf{1 1}}$ & $-3.656+28.76 \mathrm{j}$ & 0 & 0 \\
\hline $\mathbf{P}_{\mathbf{1 2}}$ & $-4.743+39.70 \mathrm{j}$ & 0 & 0 \\
\hline
\end{tabular}

\subsection{Classification results}

The databases have been built by using 8 examples of different sizes for each perfectly electric conducting target: thin wire (length: $1=1,1.3,1.5,1.7,2,2.4$, $2.5,3 \mathrm{~m}$, and radius: $\mathrm{r}=0.0025 \mathrm{~m}$ for all the wires), sphere (radius: $\mathrm{r}=0.15,0.2$, $0.28,0.3,0.35,0.4,0.45$, and $0.5 \mathrm{~m})$ and cylinder $(\mathrm{l}=0.4$ and $\mathrm{r}=0.17 \mathrm{~m}, \mathrm{l}=0.4$ and $\mathrm{r}=0.23 \mathrm{~m}, \mathrm{l}=0.5$ and $\mathrm{r}=0.15 \mathrm{~m}, \mathrm{l}=0.5$ and $\mathrm{r}=0.25 \mathrm{~m}, \mathrm{l}=0.6$ and $\mathrm{r}=0.2 \mathrm{~m}, \mathrm{l}=0.7$ and $\mathrm{r}=0.1 \mathrm{~m}, \mathrm{l}=0.8$ and $\mathrm{r}=0.19 \mathrm{~m}$, and $\mathrm{l}=1$ and $\mathrm{r}=0.15 \mathrm{~m}$ ).

Table 2: $\quad$ Classification accuracy.

\begin{tabular}{|c|c|c|c|c|c|}
\hline Classifiers & \multirow{2}{*}{$\begin{array}{l}\text { Naïve } \\
\text { Bayes }\end{array}$} & \multicolumn{3}{|c|}{ K-NN } & SVM \\
\cline { 3 - 5 } & $\mathrm{K}=1$ & $\mathrm{~K}=3$ & $\mathrm{~K}=5$ & \\
\hline $\begin{array}{c}\text { MPM in time } \\
\text { domain }\end{array}$ & $75 \%$ & $83.33 \%$ & $83.33 \%$ & $79.17 \%$ & $91.67 \%$ \\
\hline $\begin{array}{c}\text { MPM in } \\
\text { frequency } \\
\text { domain }\end{array}$ & $100 \%$ & $100 \%$ & $91.67 \%$ & $91.67 \%$ & $100 \%$ \\
\hline
\end{tabular}

A robust method for automatic target classification is presented in this work. The accuracy of the proposed method is tested on three canonical target geometries with different geometry dimensions. Results indicate that the designed classifier, which is based on the use of the Matrix Pencil method in frequency domain and different classifier, can be an efficient solution for automatic target classification. 
In this work, we used the vertical polarization in emission and we showed that the classification accuracy increases by using features extracted from the two components of the received signal.

\section{Conclusion}

In this paper, the application of Matrix Pencil method to two different cases has been demonstrated. The applications are related to cases of non-intrusive load monitoring and target classification by using UWB radar and can be easily applied to a radiated EM field from thin wire systems.

In particular, the MP method shows several advantages:

- Robustness against measurement noises.

- Less data required to identify the original signal by using reduced number of poles and residues.

- Application in the real axis (time domain) or in the complex plane (the frequency and space domains).

Different realistic case scenarios are studied and the results arising from this analysis seem to be rather promising.

\section{References}

[1] Y. Hua, "On techniques for estimating parameters of exponentially damped/undamped sinusoids in noise", Ph.D. dissertation, Syracuse University, 1987.

[2] Y. Hua and T. K. Sarkar, "Generalized pencil-of-function method for extracting poles of an EM system from its transient response", IEEE Transactions on Antennas and Propagation, AP-37, 229-234, 1989.

[3] Y. Hua and T. K. Sarkar. On the total least squares linear prediction method for frequency estimation. IEEE Transactions on Acoustics, Speech and Signal Processing, 38(12): 2186-2189, 1990.

[4] H. Najmeddine, K. El Khamlichi Drissi, C. Pasquier, C. Faure, K. Kerroum, T. Jouannet, M. Michou and A. Diop, "Smart Metering by using Matrix Pencil', 9th EEEIC conference, 16-19 May 2010, Prague, Czech Republic, 238-241.

[5] A. Diop, T. Jouannet, K. El Khamlichi Drissi and H. Najmeddine, "Method and device for the non-intrusive determination of the electrical power consumed by an installation, by analysing load transients", World Patent, WO 2010037988 A1, 8 April 2010.

[6] H. Najmeddine and K. El Khamlichi Drissi, "Advanced Monitoring with a Smart Meter", Electrical and Electronic Review, Mendeley, Vol. 86, ${ }^{\circ}$ 12, pp. 243-246, December 2010.

[7] Z. Ding J. E. Mooney and L. S. Riggs. Robust target identification in whitegaussian noise for ultra wide-band radar systems. IEEE Transactions on Antennas and Propagation, 46:18171823, 1998.

[8] M. Khodjet-Kesba, K. El Khamlichi Drissi, S. Lee, K. Kerroum, C. Faure and C. Pasquier, "Comparison of Matrix Pencil Extracted Features in Time 
Domain and in Frequency Domain for Radar Target Classification", International Journal of Antennas and Propagation, Vol. 2014, 9 pages, February 2014.

[9] C-C Chen. Electromagnetic resonances of immersed dielectric spheres. IEEE Transaction on Antennas and Propagation, 46: 1047-1083, July 1998.

[10] J.E. Mooney, Z. Ding, and L.S. Riggs, "Robust target identification in white Gaussian noise for ultra wide-band radar systems", IEEE Trans Antennas and Propagation, 46, 1817-1823, 1998.

[11] M. McClure, R. C. Qiu, and L. Carin, "On the superresolution of observables from swept-frequency scattering data," IEEE Trans. Antenna Propagat, vol. 45, 631-641, April 1997.

[12] FEKO Comprehensive Electromagnetic Solutions, User's Manual, Suite 6.0, September 2010.

[13] M. Khodjet-Kesba, K. Chahine, K. El Khamlichi Drissi, and K. Kerroum, "Comparison of Ultra-wideband Radar Target Classification Methods Based on Complex Natural Resonances", Progress In Electromagnetics Research Symposium, PIERS, Kuala-Lumpur, Malaysia, 306-309, 27-30 March, 2012. 